be used to define novel diagnostic subgroups

In another use of the Lymphochip, we have discovered 33 novel lymphoidrestricted genes. Of these, 12 were not induced during in vitro B cell activation and were restricted in expression to germinal center B cells or memory B cells. Thus, transit of B cells through the germinal center induces a differentiation event that is marked by coordinate expression of a set of late-stage B cell differentiation genes. The expression of these genes is maintained even as the B cell leaves the germinal center microenvironment and differentiates into a non-dividing memory B cell. Some novel genes were found to be differentially regulated between centroblasts and centrocytes, showing the power of this functional genomics approach to elucidate molecular changes that occur during the process of positive and negative selection in the germinal center.

Amorese, Douglas

\section{Characterization of CDNA microarrays fabricated using thermal ink jets}

\author{
M. Caren ${ }^{1}$, P. Webb ${ }^{1}$, L. Bruhn' ${ }^{1}$, D. Wiest ${ }^{1}$, D. Ilsley' ${ }^{1}$, L. DaQ uino ${ }^{1}$ \\ W. Fisher ${ }^{1}$, R. Tella ${ }^{1}$, H. Cattell ${ }^{1}$, K. Schliefer ${ }^{1}$, J. Bass ${ }^{1}$, \\ D. Roitman'1, G. Fulcrand ${ }^{1}$, R. Simon ${ }^{1}$, P. Tseng ${ }^{1}$, M. Westa1', M. \\ Bittner $^{2}$, Y. Chen', P. M eltzer'2, J. Trent ${ }^{2} \&$ D. Amorese ${ }^{1}$ \\ ${ }^{1}$ Hewlett Packard Company, Bioscience Products and Hewlett Packard Labs, \\ Palo Alto, California 94304, USA \\ ${ }^{2}$ National Human Genome Research Institute, Bethesda, M aryland, 20892, USA
}

Thermal ink jets (TIJ) have been used as a micro-delivery system for the deposition of cDNAs onto modified glass supports. We have modified TIJ heads and firing electronics, designed for the printing of ink, to form the core of a micro deposition system that efficiently, reliably and accurately deposits large numbers of cDNAs into tightly packed arrays. Data will be presented demonstrating that these arrays, generated by the TIJ process, generally perform similarly to arrays generated using conventional pen technology but offer advantages in feature quality, control of spot size and large-scale manufacturing. Quality assurance data from the drop detect and inspection systems used to qualify production runs will be presented as well as results from experiments using multiple TIJ fires to produce variable feature sizes. Lower limit of detection and two-colour competitive hybridization data collected from a new high-speed, high depth of field, autofocus scanner (under development) will also be presented.

Amundson, Sally

\section{The 'stress chip': a specialized functional genomics application for the study of genotoxic stress response}

\author{
Sally A. Amundson, M ichael Bittner, Bill Giasi, Khanh T. Do, \\ Paul M eltzer, Jeffrey Trent \& Albert J. Fomace Jr
}

$\mathrm{NIH}, \mathrm{NCl}$, Bethesda, M aryland 20892, USA

The cellular responses to ionizing radiation and other genotoxic stresses are complex and are regulated by a number of overlapping pathways. One such stress-signalling pathway is exemplified by the transcription factor $\mathrm{p} 53$, which can regulate the expression of a myriad of downstream genes in response to various stresses. We have recently demonstrated the utility of cDNA microarray hybridization to measure radiation stress-gene responses in p53 wild-type human cells. Our initial experiments also resulted in the identification of a number of new radiationresponsive genes. We have since extended these studies to a broader range of doses and timepoints, and identified a large set of genes regulated in response to ionizing radiation, as well as other stresses. These and other newly identified stress-responsive genes are being assembled in a stress-specific array, tailored for use in future genotoxic stress experiments. By focusing our efforts on a subset of responsive genes, we hope to rapidly obtain large stress-specific databases suitable for an informatics approach to analysis. Similar analytical approaches in our laboratory have previously yielded important mechanistic insights into the function of the gene GADD45, forming an important precedent for the utility of informatics analysis. The wide variation in responses observed for some stress-responsive genes in different cell lines, or in response to different genotoxic stresses, highlights the importance of cellular context to stress response, as well as the need for informatics approaches to the study of stress-specific gene response pathways.

Baker, Henry

\section{Use of global genomic expression studies to define Gcrlp as a major transcription- al activator}

\author{
M. Cecilia López \& Henry V. Baker
}

Department of M olecular Genetics and M icrobiology, Box100266, University of Florida, Gainesville, Florida 32610, USA

In Saccharomyces cerevisiae the enzymes of glycolysis make up 30-60\% of the soluble cellular protein. The first indication that glycolytic enzyme genes were coordinately regulated came with the isolation of gcrl mutants, which have severely reduced levels of most glycolytic enzymes. GCRl encodes a positive transcriptional activator, Gcrlp, that binds at the UAS elements of glycolytic enzyme genes with the assistance of Raplp. An important question concerning Gcrlp is the extent of its action in the cell. Using high-density DNA microarrays, we defined a limited set of genes that are dependent on Gcrlp. For these studies we used two isogenic strains, wild type and a gcrl mutant grown in the permissive medium of YP supplemented with glycerol and lactic acid. RNA was collected from cells during steady-state growth and used to prepare labelled cDNA. The labelled cDNA was hybridized to DNA microarrays. ORFs that displayed threefold or higher hybridization to cDNA from wild-type cells accounted for $30 \%$ of the hybridization of the total transcriptome, whereas these same ORFs accounted for only $4 \%$ of the transcriptome of the $g c r l$ mutant. We identified approximately $50 \mathrm{ORFs}$ using the threefold cutoff. These 'GCRI-dependent genes' were placed in three classes: (i) glycolytic enzyme genes; (ii) ORFs encoded by Ty elements; and (iii) genes whose expression was not previously known to be dependent on Gcrlp. Of the eight glycolytic enzyme activities known to be severely affected by $g c r l$ mutations, ten ORFs encoding seven of the activities were identified. Only PGII was not identified using the threefold cutoff; it displayed a twofold difference. In total 32 of the GCR1-dependent genes identified by microarry analysis were previously known, or were expected, to be dependent on GCR1. The expression of the remaining ORFs identified was not previously known to be dependent on GCR1. Genes encoding ribosomal proteins were not identified as GCRI dependent, suggesting that Rap1p interacts with activators other than Gcr1p at ribosomal protein gene UAS elements. In summary, a limited set of genes is dependent on GCR1 for expression, but together they compose $30 \%$ of the yeast transcriptome. The genomic expression studies described here further establish Gcr1p as one of the most potent transcriptional activators. 